Produto \& Produção, vol. 11, n. 2, p. 104 - 119, jun. 2010

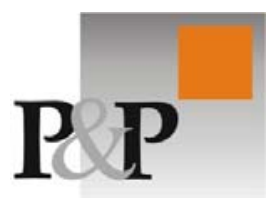

\title{
Processo de Validação Interna de um Questionário em uma Survey Research Sobre ISO 9001:2000
}

\author{
Marcelo Hoss \\ Programa de Pós-Graduação em Engenharia de Produção - UFRGS \\ Carla Schwengber ten Caten \\ Programa de Pós-Graduação em Engenharia de Produção - UFRGS
}

\begin{abstract}
RESUMO
Muitos trabalhos desenvolvidos na literatura sobre ISO 9001:2000 utilizam a survey research (pesquisa de levantamento) como forma de obtenção de dados para suas pesquisas. Esta metodologia tenta descobrir relações entre variáveis dentro de uma população a partir de uma amostra utilizando questionários como instrumentos de medição. Contudo, antes de realizar a validação externa (inferências), é necessário verificar a validade interna do questionário, ou seja, se o instrumento mede o que está proposto a medir, já que a falta deste processo pode comprometer os resultados encontrados pelos pesquisadores. Assim, este trabalho tem como objetivo apresentar um processo de validação interna de um questionário aplicado a empresas certificadas ISO 9001:2000 e não certificadas. Os passos adotados foram: validação de conteúdo, análise fatorial exploratória, validação de construção, verificação de confiabilidade e análise detalhada dos itens.
\end{abstract}

Palavras-chave: pesquisa de levantamento; validade interna de questionário; ISO 9001:2000

\section{INTRODUÇÃO}

A decisão de procurar um sistema de gestão da qualidade como a ISO 9001:2000 ${ }^{1}$ e efetivá-lo com a certificação pode proporcionar consideráveis benefícios em longo prazo para as organizações (NQA, 2006). Como conseqüência, a ISO 9001:2000 vem se tornando cada vez mais popular no meio empresarial uma vez que o número de certificados expedidos no mundo aumenta a cada ano (ISO, 2007). Este fato atraiu a atenção do meio científico, que tenta verificar a efetividade

\footnotetext{
${ }^{1}$ Existe uma versão mais recente da norma aprovada no final de 2008. Entretanto, como as mudanças não foram significativas entre as normas e a coleta de dados deste trabalho foi realizada antes desta data, preferiu-se utilizar a versão 2000 no texto.
} 
da ISO 9001 nas empresas. Neste sentido, estudos têm sido desenvolvidos em países como Hong Kong, EUA, Austrália, Singapura, Espanha, Dinamarca (LEUNG et al., 1999; TERLAAK; KING, 2005; TERZIOVSKI et al., 1997; NAVEH; MARCUS, 2005; HERAS et al., 2002a,b; HÄVERSJÖ, 2000), entre outros.

Esses trabalhos utilizam a survey research (pesquisa de levantamento) para identificar quais são as motivações que levam as organizações a obterem a certificação, quais são as práticas de gestão adotadas ou para realizar comparações de desempenho das organizações certificadas e não certificadas. Contudo, os resultados encontrados nos estudos muitas vezes podem ser questionados metodologicamente, pois os instrumentos de medição (questionários) não passam por nenhum processo de validação.

A pesquisa de levantamento é uma metodologia que tenta descobrir relações entre variáveis dentro de uma população. Há duas principais categorias para esta metodologia: o censo, em que se pesquisa toda a população, e o levantamento amostral, no qual se investiga apenas uma parcela da população. O pesquisador, neste segundo tipo, generaliza a população através de uma amostra. Destacam-se três instrumentos de medição para este tipo de pesquisa: entrevistas por telefone, face a face e envio de questionário (correio eletrônico ou convencional). Independentemente do instrumento, a metodologia requer que dois tipos de validação sejam realizados: a validação interna, que se refere ao quão bem o instrumento mede o que está proposto a medir e a validação externa, que se relaciona com os testes de hipóteses a partir dos dados amostrais com o objetivo de se inferir uma situação provável na população (GIUFFRE, 1997a,b).

Assim, este estudo tem como objetivo apresentar um processo de validação interna de um questionário de uma pesquisa de levantamento amostral realizado com empresas certificadas e não certificadas ISO 9001:2000 da cadeia coureirocalçadista brasileira. Um convite foi enviando por e-mail para cada elemento da amostra para preencher um questionário via internet sobre as práticas de gestão adotadas pela empresa. A seguir é apresentada a revisão bibliográfica para o processo de validação interna do mesmo.

\section{REVISÃO BIBLIOGRÁFICA}

A validação interna atribui qualidade ao instrumento de medição. Uma boa mensuração, segundo Hubley e Zumbo (1996), deve ter duas características essenciais: confiabilidade e validade. A confiabilidade é sinônimo de consistência, estabilidade e previsibilidade. Já a validade tem um sentido de acuracidade, autenticidade e veracidade. Apesar de ambas apresentarem conceitos diferentes, elas estão relacionadas da seguinte forma: uma observação pode ser confiável sem ser válida, mas não válida sem antes ser confiável. Em outras palavras, confiabilidade é necessária, mas não condição suficiente para a validade. 0 presente estudo identificou os testes mais utilizados de validade e confiabilidade nos questionários dos trabalhos de Saraph et al. (1989), Ahire et al. (1996), Black e Porter (1996), Rahman (2001), Kaynak (2003) e Conca et al. (2004). Nas próximas seções são discutidos os seguintes tópicos: questionário, validade de conteúdo, análise fatorial, validade de construção, confiabilidade e análise detalhada de itens. 


\subsection{Questionário}

O questionário, de acordo com Gil (1995), é uma técnica de investigação composta por um número determinado de questões apresentadas por escrito a um grupo de pessoas, tendo como objetivo o conhecimento de suas opiniões. A utilização dessa técnica propicia vantagens como a possibilidade de atingir grande número de respondentes mesmo em áreas geográficas distantes, a flexibilidade de respondê-lo quando se julgar mais conveniente e a não exposição dos pesquisados à influência das opiniões do entrevistador. Contudo, os questionários podem apresentar resultados não esperados, já que os itens podem ter significados diferentes para cada respondente. Outro problema é a limitação da quantidade de questões, pois questionários muito extensos apresentam alta probabilidade de não serem respondidos.

\subsection{Validade de conteúdo}

Para Haynes et al. (1995), validade de conteúdo é o grau no qual os elementos constitutivos de um instrumento de mensuração são representativos e relevantes para o conceito a ser avaliado. Ou seja, ao se construir um questionário, este deve contemplar todos os fatores do fenômeno que se deseja medir. Os autores sugerem um guia com pontos para a validação de conteúdo, tais como: definir o domínio de construção, geração, avaliação e correção do conteúdo por especialistas, e emprego de outras análises para refinamento do instrumento.

\subsection{Análise fatorial}

Um questionário é composto por um número de questões (itens) que tentam traduzir um conceito geral de uma dimensão. Freqüentemente, os itens são agrupados em fatores para explicar sub-conceitos (constructos) dentro de uma dimensão. Assim, para validar um questionário é necessário verificar se os itens são suscetíveis a serem agrupados. Desta forma, apresenta-se primeiramente a análise fatorial para posteriormente apresentar as demais técnicas de validação e confiabilidade. A análise fatorial foi empregada de duas formas neste trabalho. A primeira foi exploratória para extrair os fatores da dimensão em estudo; a segunda, como confirmatória, para verificar a unidimensionalidade dos fatores na validade de construção.

Conceitualmente, a análise fatorial é uma técnica estatística multivariada que estuda correlações entre um grande número de variáveis agrupando-as em fatores. A análise fatorial exploratória (AFE), ao contrário da confirmatória, não define restrições ou número de componentes a serem extraídos. Assim, uma vez que os fatores estajam determinados e interpretados, estes podem descrever os dados em um número menor de conceitos do que as variáveis individuais (HAIR et al., 1995).

A demonstração matemática a seguir foi baseada em Johnson e Wichern (1982). O vetor observável $X$ (item do questionário) com $p$ variáveis (número de itens de uma dimensão) possui média $\mu$ e matriz de covariância $\Sigma$. O modelo de fatores 
postula que $X$ é linearmente dependente de algumas variáveis aleatórias não observadas F1, F2, ... Fm, chamadas de fatores comuns, e a fontes adicionais de variação $\varepsilon 1, \varepsilon 2, \ldots, \varepsilon p$, chamados de erros ou fatores específicos. O modelo da análise fatorial é:

$$
\begin{aligned}
& X_{1}-\mu_{1}=\ell_{11} F_{1}+\ell_{12} F_{2}+\ldots+\ell_{13} F_{3}+\varepsilon_{1} \\
& X_{2}-\mu_{2}=\ell_{21} F_{1}+\ell_{22} F_{2}+\ldots+\ell_{23} F_{3}+\varepsilon_{2} \\
& \vdots \\
& X_{p}-\mu_{p}=\ell_{p 1} F_{1}+\ell_{p 2} F_{2}+\ldots+\ell_{p 3} F_{3}+\varepsilon_{p}
\end{aligned}
$$

ou, em notação vetorial:

$$
X_{(p \times 1)}-\mu_{(p \times 1)}=L_{(p \times m)} F_{(m \times 1)}+\varepsilon_{(p \times 1)} .
$$

O coeficiente $\ell_{i j}$ é chamado de peso, carga ou carregamento (loading) da variável i no fator $\mathrm{j}$, resultando assim na matriz $\mathrm{L}$. Devido ao grande número de variáveis não observadas para serem determinadas, é necessário que se façam algumas considerações. Os vetores $F$ e $\varepsilon$ devem ser independentes, ou seja, $\operatorname{Cov}(\varepsilon$, $F)=0$, onde $E(F)=0, \operatorname{Cov}(F)=I$ e $E(\varepsilon)=0, \operatorname{Cov}(\varepsilon)=\Psi$, tal que $\Psi$ é uma matriz diagonal. O modelo ortogonal de fatores implica que:

$$
\begin{gathered}
\sum=\operatorname{Cov}(X)=L L^{\prime}+\Psi \\
\operatorname{Var}\left(X_{i}\right)=\ell_{i 1}^{2}+\ldots+\ell_{i m}^{2}+\psi_{i} \\
\operatorname{Cov}\left(X_{i}, X_{k}\right)=\ell_{i 1} \ell_{k 1} \ldots+\ell_{i m} \ell_{k m i} \\
\operatorname{Cov}(X, F)=L \\
\operatorname{Cov}\left(X_{i}, F_{j}\right)=\ell_{i j} .
\end{gathered}
$$

Existem vários métodos de estimação para $\Sigma$, Contudo o método mais utilizado é dos componentes principais. Este especifica que a matriz de covariâncias $\mathrm{S}$, um estimador da matriz de covariâncias $\Sigma$, possua pares de autovalores $\mathrm{e}$ autovetores $\left(\hat{\lambda}_{1}, \hat{e}_{1}\right), \ldots,\left(\hat{\lambda}_{p}, \hat{e}_{p}\right)$ onde $\hat{\lambda}_{1} \geq \hat{\lambda}_{12} \geq \ldots \hat{\lambda}_{p}$. O autovalor representa a variância total explicada por cada fator, sendo o número de fatores $\mathrm{m}$ menor ou igual ao número de variáveis p. A matriz estimada dos fatores carregados $\{\tilde{\ell}{ }\}$ é dada por:

$$
\tilde{L}=\left[\sqrt{\hat{\lambda}_{1}} \hat{e}_{1} ; \sqrt{\hat{\lambda}_{2}} \hat{e}_{2} ; \ldots ; \sqrt{\hat{\lambda}_{m}} \hat{e}_{m}\right] \text {. }
$$

As estimativas das variâncias específicas são geradas pelos elementos da diagonal da matriz $S-\tilde{L} \tilde{L}^{\prime}$. Assim,

$$
\Psi=\left[\begin{array}{cccc}
\tilde{\psi}_{1} & 0 & \ldots & 0 \\
0 & \tilde{\psi}_{2} & \ldots & 0 \\
\vdots & \vdots & \ddots & \vdots \\
0 & 0 & \ldots & \tilde{\psi}_{p}
\end{array}\right] ; \quad \tilde{\psi}_{i}=s_{i i}-\sum_{j=1}^{m} \tilde{\ell}_{i j}^{2} .
$$

A comunalidade, que é a quantidade da variância que uma variável compartilha com as outras variáveis, pode ser estimada como:

$$
\tilde{h}_{i}^{2}=\tilde{\ell}_{i 1}^{2}+\tilde{\ell}_{i 2}^{2}+\ldots+\tilde{\ell}_{i m}^{2} \text {. }
$$

A estimativa inicial de $S$ é obtida utilizando a matriz de correlação $R$. A proporção da variância total da amostra devido ao fator $\mathrm{j}$ pode ser calculada por 
$\hat{\lambda}_{j} / p$, ou seja, o autovalor dividido pelo número máximo de fatores. Normalmente, utiliza-se o critério da raiz latente para a determinação do número de fatores, onde o número de fatores corresponde ao número de autovalores maiores ou iguais a um.

Após a extração dos fatores, a matriz de carregamento sofre uma rotação onde esta é multiplicada por uma matriz ortogonal para se obter uma interpretação mais fácil dos fatores. O método mais popular de rotação é o critério de Kaiser, denominado VARIMAX, que busca a alocação mais simplificada dos fatores encontrando grupos de carregamento significativos e negligenciáveis. Após a alocação das variáveis em cada fator, pode-se criar uma escala aditiva. Esta escala é a média das variáveis significativas do fator. A criação de uma escala aditiva é conveniente por reduzir a variabilidade, já que agrupa variáveis que acabam medindo um mesmo conceito.

A adequação do modelo à consideração de que a amostra provém de uma população normal, um requisito para análise fatorial, conduz ao teste de Bartlett. $\mathrm{O}$ teste de esfericidade de Bartlett rejeita a hipótese de que as variáveis não sejam correlacionadas na população a um determinado $\alpha$, se:

$$
(n-1-(2 p+4 m+5) / 6) \ln \frac{\tilde{L} \tilde{L}^{\prime}+\hat{\Psi}}{S_{n}}>\chi_{\left.[p-m)^{2}-p-m\right] / 2}^{2}(\alpha) .
$$

A medida de adequação da amostra (MAA), ou teste de Kaiser-Meyer-Olkin (KMO), é um indicador utilizado para examinar a conveniência da execução de uma análise fatorial. É calculado tanto para toda a matriz de correlação como para cada variável.

\subsection{Validade de construção}

A validação de construção de um questionário sempre é necessária quando não há nenhum critério de mensuração direto definido para o atributo que se deseja medir. Se uma dimensão é composta por mais de um fator, o problema surge na definição da parcela de variância destinada a cada mensuração indireta, ou seja, a cada fator (CRONBACH; MEEHL, 1955). Uma quantidade de itens de uma dimensão pode ser agrupada em subdimensões, chamados de fatores. Contudo, para se poder alocar uma parcela da variância dos itens a cada fator deve-se, primeiramente, checar se os fatores são unidimensionais. A unidimensionalidade de um fator significa que não é possível extrair outros fatores deste. Para isso, utiliza-se a análise fatorial confirmatória como método de validade de construção (BAGOZZI et al., 1991). 
O erro aleatório está presente em qualquer mensuração científica e acaba por reduzir a precisão das mesmas. Desta forma, a confiabilidade refere-se à precisão da mensuração independente do que é medido. A consistência interna, como medida de confiabilidade de um questionário, indica quão diferente os itens medem o mesmo conceito (NUNNALLY, 1978). O estimador mais utilizado para a consistência interna é o alfa de Cronbach (PETERSON, 1994) e sua formulação é dada por:

$$
\alpha=\left(\frac{k}{k-1}\right)\left(1-\sum_{i=1}^{k} \sigma_{i}^{2} / \sigma_{s}^{2}\right),
$$

onde k é o número de pontos de uma escala, $\sigma_{i}^{2}$ é a variância do item i e $\sigma_{s}^{2}$ é a variância da escala. Cabe ressaltar a observação de Cortina (1993) de que o coeficiente alfa é útil para a estimativa da confiabilidade em um caso particular no qual a variância específica do fator é unidimensional. Se a validade de construção sugerir a existência de um único fator, então valores altos de alfa de Cronbach podem ser usados para concluir que o conjunto de itens que compõe o fator é unidimensional e que grande parte da variância é atribuída ao seu comportamento.

\subsection{Análise detalhada de itens}

A análise detalhada de itens é um método para avaliação da correta alocação de itens nos fatores. Considera-se a correlação de cada item com cada fator para verificar se o item pertence ou não ao fator em que foi alocado (NUNNALLY, 1978). Se um item pertencente a um fator tiver uma correlação maior com outro fator ao qual não foi designado, este item deveria ser reposicionado ou eliminado (SARAPH et al., 1989).

\section{METODOLOGIA}

A Figura 1 apresenta a metodologia adotada neste estudo. A validação interna apresenta passos (caixas com linhas cheias) que foram executados seqüencialmente e resultaram em um produto final (caixa com linhas tracejadas). Este produto final permite a realização da validade externa (checar relações hipotetizadas) de acordo com os objetivos de cada pesquisa. Cabe ressaltar que a validade de conteúdo, apesar de fazer parte da validação interna, foi operacionalizada na construção do questionário. Desta forma, os pontos que caracterizam a validade de conteúdo apresentados por Haynes et al. (1995) são discutidos a seguir.

O questionário foi dividido em duas partes: a primeira caracterizou as organizações e a segunda avaliou a dimensão "Práticas de Gestão" das organizações. Na primeira parte, foi perguntado ao respondente sobre a sua função e área de atuação dentro da empresa. Sobre a empresa foi questionado o tipo de atividade produtiva, estado federativo onde a unidade está situada, número de 
funcionários e condição da unidade fabril em relação à certificação ISO 9001:2000. Já na segunda parte, questionou-se sobre as práticas de gestão que a empresa adota (21 questões) baseadas nos oito princípios da ISO 9000:20002 (ABNT, 2001). Estes princípios são: foco no cliente, liderança, envolvimento de pessoas, abordagem de processo, abordagem sistêmica para a gestão, melhoria contínua, abordagem factual para a tomada de decisão, benefícios mútuos nas relações com fornecedores. As questões foram extraídas a partir dos seguintes trabalhos: Powell (1995), Black e Porter (1996), Buttle (1997), Leung et al. (1999), Wilson e Collier (2000), Escanciano et al. (2001), Rahman (2001), Sohal e Terziovski (2000), Singels et al. (2001), Gotzamani e Tsiotras (2002), Chow-Chua et al. (2003), Kaynak (2003) e Conca et al. (2004). Estas questões foram aleatorizadas para dispersar o contágio dos princípios nos possíveis fatores que possam surgir (BLACK; PORTER, 1996). A escala utilizada foi do tipo Likert de 5 pontos, onde (1) é "fortemente discorda" e (5) é "fortemente concorda".

Além disso, o questionário possuiu outras características que convergem para uma validade de conteúdo. No cabeçalho, foi realizada uma apresentação sobre objetivos da pesquisa e respectivas instruções de preenchimento para familiarizar o respondente com o questionário. Da mesma forma, o texto das questões foi simplificado com a revisão de sete pesquisadores que verificaram erros gramaticais, exaustividade e complexidade de entendimento. A construção do questionário disponibilizado na página da internet foi realizada com letras grandes e de diferentes cores para facilitar a visualização. $O$ questionário pôde ser preenchido, em testes realizados, em menos de 15 minutos em função da agilidade fornecida pelas questões fechadas. $O$ número de questões elaboradas para cada um dos oito princípios variou entre 2 a 4 itens. Finalmente, foram empregados outros tipos de análises de validade e confiabilidade para o refinamento do instrumento de avaliação.

\footnotetext{
${ }^{2}$ Cabe ressaltar as diferenças entre as normas. A ISO 9000 é norma que apresenta as definições, princípios e vocabulário relacionados ao sistema de gestão da qualidade. Já a norma ISO 9001 apresenta o conjunto de requisitos que devem ser atendidos para se obter a certificação. Muitas vezes o termo ISO 9000 também é usado para designar a família de normas ISO 9000, ISO 9001 e ISO 9004.
} 


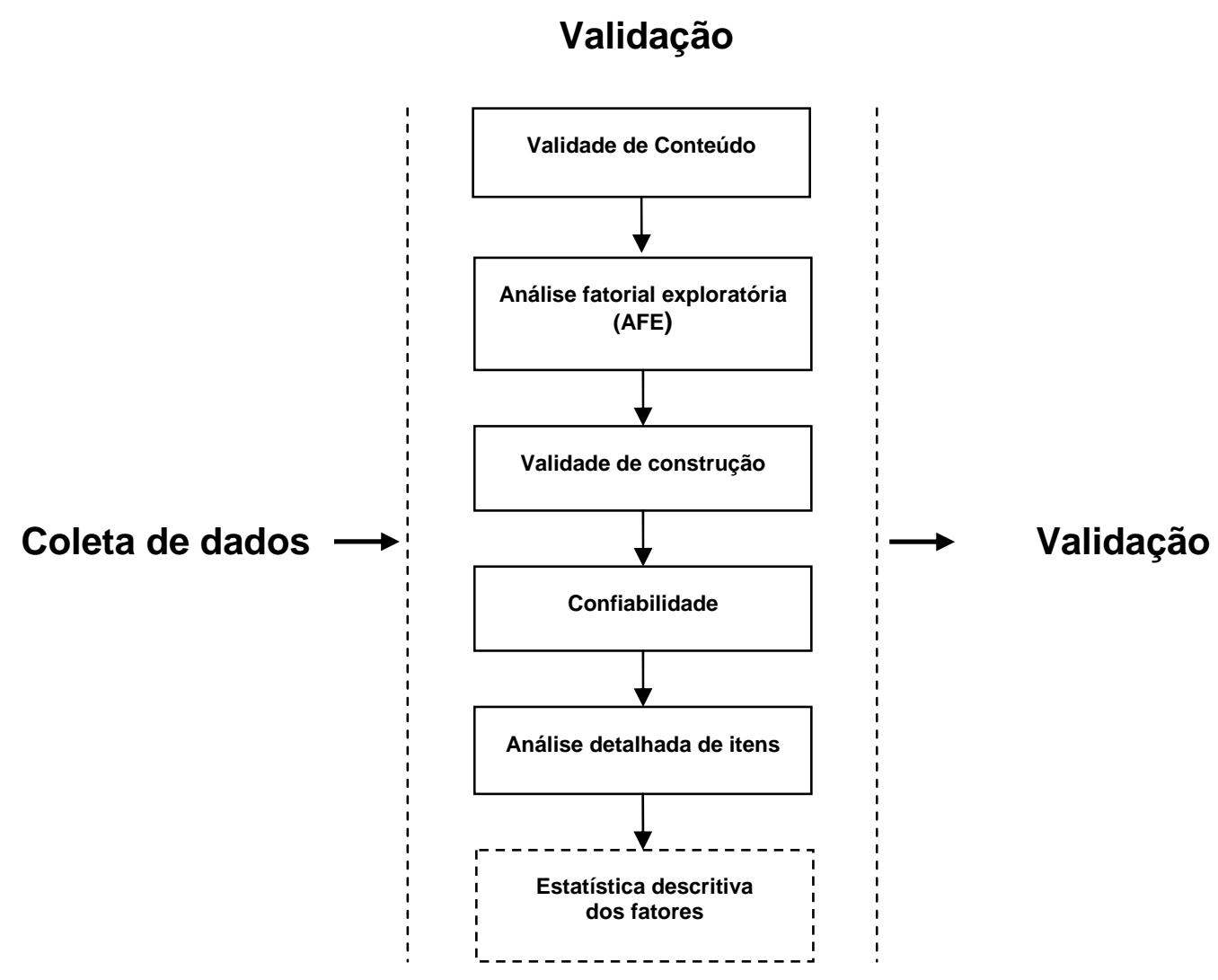

Figura 1- Processo de validação interna da metodologia de levantamento de pesquisa amostral

\subsection{Coleta de dados}

A população alvo deste estudo abrangeu as empresas da cadeia coureirocalçadista brasileira. Identificaram-se as associações que representam os setores produtivos da cadeia (componentes e produtos químicos, curtumes, calçados e máquinas e equipamentos). A partir das associações, obtiveram-se listas de empresas e seus respectivos e-mails para contato. $O$ total de empresas que compuseram a população foi de 1253. Cada empresa recebeu um e-mail convite semanal durante um mês para o preenchimento de um questionário via internet.

Obtiveram-se 130 respostas consideradas como válidas (taxa de resposta $10,4 \%)$. A amostra foi composta por $65,38 \%$ de empresas com até 99 funcionários, $25,38 \%$ entre 100 e 499 e $9,24 \%$ maiores que 500 funcionários. Além disso, 45 das 130 empresas possuem a certificação ISO 9001:2000. 


\subsection{Resultados da análise fatorial exploratória}

Nesta seção foram extraídos e validados os fatores que melhor representam as "Práticas de Gestão" de uma organização. Para realizar uma boa AFE, a amostra deve ter um tamanho apropriado. Hair et al. (2005) recomendam um tamanho mínimo da amostra cinco vezes maior que o número de itens a serem avaliados. Como o questionário é composto por 21 itens e a amostra possui 130 observações, tem-se um indicativo positivo da adequação do tamanho da amostra.

As considerações acerca da análise fatorial são mais conceituais do que propriamente estatísticas. A falta de normalidade, homocedacidade ou linearidade dos dados somente diminuem as correlações observadas. Além disso, algum grau de multicolinearidade é desejado, pois o objetivo é identificar correlações entre as variáveis. Contudo, deve-se assegurar que a matriz de dados possua correlações suficientes que justifiquem a análise fatorial (HAIR et al., 2005). Um indicativo para a realização da análise fatorial é que apenas 17 correlações, de um total de 210, são menores que 0,3 e apenas uma delas não é significativa a 0,001. Hair et al. (2005) comentam que a medida de adequação da amostra (MAA) também pode ser utilizada para verificar se análise fatorial seria aplicável. A MAA pode variar de 0 até 1 indicando quanto cada variável é predita sem erros por outras variáveis. A MAA pode ser utilizada tanto para variáveis individuais quanto para o conjunto de variáveis da amostra (KMO), sendo que valores aceitáveis deveriam ser maiores a 0,5 . O KMO deste estudo resultou no valor de 0,929. Além disso, não houve nenhum valor individual de MAA menor que 0,5. O teste de esfericidade de Bartlett foi significativo demonstrando a normalidade dos dados. Desta forma, a realização da AFE para a dimensão de práticas de gestão foi apropriada.

Pelo critério da raiz latente, foram extraídos três fatores que explicam 62,67\% da variância total da dimensão. Este cálculo é realizado pela soma dos autovalores extraídos $(13,16)$ dividido pelo número máximo de fatores possíveis a serem extraídos (21); ou seja, no limite cada um dos 21 itens poderia ser considerado como um fator. Hair et al. (2005) comentam que uma extração de $60 \%$ da variância seria aceitável para pesquisas sociais exploratórias e descritivas como neste estudo. A extração de fatores pelo critério VARIMAX não forneceu um padrão entendível de alocação de itens aos fatores. Desta forma, foi necessária a rotação dos fatores para facilitar o entendimento de cada um destes. Os carregamentos dos itens rotacionados podem ser visualizados na Tabela 1. O carregamento indica o grau de correspondência entre o item e o fator. Quanto maior o seu módulo, maior é a representatividade do item no fator. Apesar de não ter uma ênfase estatística, mas sim prática, adota-se que carregamentos maiores que o módulo de 0,5 sejam significativos, e maiores que o módulo de 0,3 sejam aceitáveis (HAIR et al., 2005). Uma vez que os itens estiverem alocados aos seus respectivos fatores, é possível checar as comunalidades. Valores menores que 0,5 não são apropriados, mostrando que a solução não extraiu a variância do item suficientemente para alocá-lo corretamente a um determinado fator. Verifica-se na Tabela 1 que todos os itens tiveram comunalidade maior que 0,5 , exceto "Trabalho em equipe nas melhorias". 
A validade de construção verifica a unidimensionalidade dos fatores utilizando a análise fatorial confirmatória. Na Tabela 2 observa-se que os fatores que sofreram a rotação apresentaram-se unidimensionais. Entretanto, a validade de construção pode ser considerada como redundante caso seja utilizada a raiz latente como critério de extração dos fatores. Neste caso, como o ponto de corte que define o número de fatores é especificado com base nos autovalores maiores ou iguais a um, a extração somente poderia fornecer fatores unidimensionais.

\subsection{Resultados da confiabilidade}

Confirmada a unidimensionalidade, pôde-se realizar a análise de confiabilidade para concluir que o conjunto de itens que compõe o fator é unidimensional e que grande parte da variância é atribuída ao seu comportamento. Nunnally (1978) recomenda um nível mínimo de 0,7 para o alfa de Cronbach. Hair et al. (2005) comentam que para pesquisas exploratórias, o alfa pode ser reduzido para 0,6 . A Tabela 1 apresenta os alfas dos fatores. Os fatores apresentaram valores de alfas maiores do que o recomendado por ambos os autores. Nota-se que, apesar do item "Trabalho em equipe nas melhorias" apresentar uma comunalidade abaixo do recomendado, caso este fosse eliminado, o alfa do fator "Gestão por processos" não aumentaria. Assim, optou-se por não eliminá-lo. 
Tabela 1 - Alocação dos itens de Práticas de Gestão

\begin{tabular}{|c|c|c|c|c|c|c|c|c|}
\hline \multirow{3}{*}{$\begin{array}{c}\text { Princípios } \\
\text { ISO 9000:2000 }\end{array}$} & \multirow{3}{*}{ Itens de Práticas de Gestão } & \multicolumn{3}{|c|}{ Cargas nos fatores após rotação } & \multirow{3}{*}{ 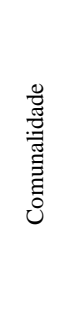 } & \multirow{2}{*}{\multicolumn{3}{|c|}{ Alfa do fator se item eliminado }} \\
\hline & & \multirow{2}{*}{ 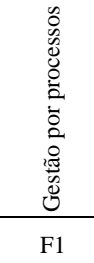 } & \multirow{2}{*}{ 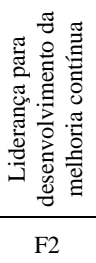 } & \multirow{2}{*}{ 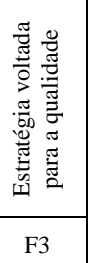 } & & & & \\
\hline & & & & & & F1 & F2 & F3 \\
\hline \multirow{2}{*}{ Foco no cliente } & Medição satisfação clientes & 0,761 & & & 0,654 & 0,907 & & \\
\hline & Melhoria produto devido reclamações & & & 0,646 & 0,637 & & & 0,710 \\
\hline \multirow{2}{*}{ Liderança } & Gerentes conduzem melhorias & & 0,470 & & 0,500 & & 0,894 & \\
\hline & Gerentes incentivam staff nas melhorias & & 0,711 & & 0,711 & & 0,880 & \\
\hline \multirow{2}{*}{$\begin{array}{l}\text { Envolvimento de } \\
\text { pessoas }\end{array}$} & Trabalho em equipe nas melhorias & 0,464 & & & 0,477 & 0,917 & & \\
\hline & Recompensa pelas melhorias & & & 0,674 & 0,603 & & & 0,725 \\
\hline \multirow{3}{*}{$\begin{array}{l}\text { Abordagem de } \\
\text { processo }\end{array}$} & Atividades documentadas & 0,822 & & & 0,741 & 0,905 & & \\
\hline & Rastreabilidade dos produtos & 0,751 & & & 0,615 & 0,911 & & \\
\hline & Revisão de projetos & 0,505 & & & 0,500 & 0,916 & & \\
\hline \multirow{3}{*}{$\begin{array}{l}\text { Abordagem } \\
\text { sistêmica para a } \\
\text { gestão }\end{array}$} & Estratégia voltada para clientes & & & 0,669 & 0,687 & & & 0,688 \\
\hline & Auto-avaliação pontos fracos e fortes & 0,808 & & & 0,809 & 0,899 & & \\
\hline & Funcionários conhecem objetivos & & 0,601 & & 0,662 & & 0,883 & \\
\hline \multirow{4}{*}{ Melhoria contínua } & Cultura melhoria contínua & & 0,703 & & 0,658 & & 0,885 & \\
\hline & Programa de redução de desperdícios & & 0,673 & & 0,607 & & 0,889 & \\
\hline & Treinamento em qualidade & 0,600 & & & 0,558 & 0,912 & & \\
\hline & Programa de ação corretiva & 0,628 & & & 0,686 & 0,908 & & \\
\hline \multirow{2}{*}{$\begin{array}{l}\text { Abordagem factual } \\
\text { para a tomada de } \\
\text { decisão }\end{array}$} & Utilização de ferramentas da qualidade & 0,729 & & & 0,633 & 0,908 & & \\
\hline & Coleta dados de mercado & & 0,481 & & 0,526 & & 0,893 & \\
\hline \multirow{3}{*}{$\begin{array}{l}\text { Benefícios mútuos } \\
\text { nas relações com } \\
\text { fornecedores }\end{array}$} & Incentivo os fornecedores para melhorias & & 0,796 & & 0,668 & & 0,894 & \\
\hline & Desenvolvimento de estratégias conjuntas & & 0,621 & & 0,600 & & 0,887 & \\
\hline & Qualidade critério de escolha de fornecedor & & & 0,783 & 0,629 & & & 0,736 \\
\hline Autovalor & & 5,41 & 4,48 & 3,27 & 13,16 & & & \\
\hline \% de variância & & 25,78 & 21,31 & 15,59 & 62,67 & & & \\
\hline Alfa do fator & & & & & & 0,919 & 0,901 & 0,769 \\
\hline
\end{tabular}


A próxima etapa da validação foi a análise detalhada de itens. Contudo, para realizar esta análise foi necessário criar uma escala aditiva que representasse cada fator. Esta escala é a média dos itens significativos do fator. Deste modo, a análise detalhada de itens verifica se os itens foram apropriadamente designados aos seus respectivos fatores. Se a correlação do item for maior com a escala do fator em que foi alocado, este deveria ser mantido no fator. Por exemplo, o item "Trabalho em equipe nas melhorias" foi designado ao fator "Envolvimento de pessoas" e caso este item possuísse uma correlação maior com o fator "Gestão por processos", então este pode ter sido alocado incorretamente. Verificou-se que todos os itens foram alocados corretamente.

Realizadas as análises de validação interna é possível nomear os fatores e interpretá-los. Black e Porter (1996) ressaltaram certas dificuldades em nomear os fatores empíricos relacionados à gestão da qualidade. Os autores comentam que os agrupamentos dos itens realizados pela AFE são mais complexos no seu entendimento do que aqueles agrupados por meios não empíricos como acontece nos prêmios da qualidade americano (Malcolm Baldrige National Quality Malcolm Program) e europeu (European Foundation for Quality Management) ou como na própria ISO 9000:2000. Neste estudo, cada fator recebeu um nome de acordo com a composição dos itens mais representativos em cada fator, ou seja, aqueles que se apresentavam em maior número de conceitos similares.

O primeiro fator da dimensão "Práticas de gestão" foi nomeado como "Gestão por processos". Este fator representa o gerenciamento da organização transformando entradas (insumos) em saídas (produtos). O segundo fator "Liderança para o desenvolvimento da melhoria contínua" apresenta a liderança que deve ser exercida na organização. Os líderes são responsáveis pela coordenação, pela participação, pelo incentivo e pela tomada de decisão para desenvolver a organização utilizando o ciclo da melhoria contínua. O último fator desta dimensão é a "Estratégia voltada para a qualidade". Este fator define o foco da organização nas questões da qualidade como a escolha criteriosa de fornecedores e a busca pela satisfação de clientes e de funcionários.

Tabela 2 - Unidimensionalidade dos fatores de prática de gestão

\begin{tabular}{|l|c|c|c|}
\hline \multicolumn{1}{|c|}{ Fatores } & KMO & Autovalor & $\begin{array}{c}\% \text { da } \\
\text { variância } \\
\text { explicada }\end{array}$ \\
\hline Gestão por processos & 0,921 & 5,48 & 60,93 \\
\hline Liderança para o desenvolvimento da melhoria contínua & 0,920 & 4,77 & 59,60 \\
\hline Estratégia voltada para a qualidade & 0,709 & 2,45 & 61,13 \\
\hline
\end{tabular}

\subsection{Estatística descritiva dos fatores}

Como produto final da validação interna de um questionário obtém-se a estatística descritiva dos fatores (Tabela 3). Ou seja, a partir do desdobramento da dimensão de "Práticas de Gestão" em três fatores e suas respectivas nomeações é 
possível ter conhecimento do desempenho, por exemplo, das organizações certificadas e não certificadas em cada fator. Assim, a estatística descritiva pode subsidiar o processo de validação externa que consiste no teste inferencial das hipóteses levantadas em função dos objetivos de cada pesquisa.

Tabela 3 - Estatística descritiva dos fatores

\begin{tabular}{|l|l|c|c|c|c|c|}
\hline \multicolumn{1}{|c|}{ Fatores } & Organizações & $\mathrm{N}$ & Mínimo & Máximo & Média & $\begin{array}{c}\text { Desvio- } \\
\text { padrão }\end{array}$ \\
\hline \multirow{2}{*}{$\begin{array}{l}\text { Gestão por } \\
\text { processos }\end{array}$} & Certificadas & 45,0 & 2,78 & 5,00 & 4,65 & 0,45 \\
\cline { 2 - 7 } & Não-certificadas & 85 & 1,00 & 5,00 & 3,47 & 0,86 \\
\hline $\begin{array}{l}\text { Liderança para o } \\
\text { desenvolvimento da } \\
\text { melhoria contínua }\end{array}$ & Certificadas & 45 & 2,75 & 5,00 & 4,44 & 0,63 \\
\cline { 2 - 7 } & Não-certificadas & 85 & 1,63 & 5,00 & 3,87 & 0,79 \\
\hline \multirow{2}{*}{$\begin{array}{l}\text { Estratégia voltada } \\
\text { para a qualidade }\end{array}$} & Certificadas & 45 & 3,00 & 5,00 & 4,43 & 0,52 \\
\cline { 2 - 7 } & Não-certificadas & 85 & 1,50 & 5,00 & 4,06 & 0,76 \\
\hline
\end{tabular}

\section{CONCLUSÕES}

Estudos na área de gestão da qualidade como, por exemplo, sobre a certificação ISO 9001:2000, têm lançado mão da metodologia de levantamento de pesquisa para inferir resultados sobre a população a partir da coleta de um conjunto de dados de uma amostra. Contudo, muitos trabalhos não apresentam nenhum processo de validação dos instrumentos de medição utilizados, comprometendo, assim, os resultados encontrados.

Este trabalho teve como objetivo apresentar um processo de validação de um questionário quando se utiliza a pesquisa de levantamento. Foi utilizado como caso um questionário sobre as "Práticas de Gestão", baseadas nos oito princípios da ISO 9000:2000, adotadas nas organizações da cadeia coureiro-calçadista brasileira. O processo de validação do questionário, chamado de validação interna, foi apresentado nos seguintes passos: validação de conteúdo, análise fatorial exploratória, validação de construção, verificação de confiabilidade e análise detalhada dos itens.

Após a validação interna, é possível nomear os fatores que pode transmitir um conceito resultante do agrupamento dos itens. A dimensão "Práticas de gestão" foi composta por três fatores. A "Estratégia voltada para a qualidade" pode ser utilizada na organização como orientação para a "Gestão por processos" que por sua vez necessita da atuação de uma "Liderança para o desenvolvimento da melhoria contínua". Neste momento, o pesquisador está apto a dar continuidade ao estudo, uma vez que o instrumento de mensuração passou por um processo de validação interna. 


\begin{abstract}
Many papers on ISO 9001:2000 available in the literature apply survey research as a methodology to obtain data from a sample. The approach in this paper attempts to discover relationships between variables within a population using questionnaires as measuring instruments. However, before carrying out the external validity analyses (inferences), it is necessary to verify the internal validity of the questionnaire, i.e., whether the instrument measures what is supposed to measure since the lack of a validation process could compromise the results found by researchers. Thus, this study aims to present an internal validity process of a questionnaire applied to certified and non-certified ISO 9001:2000 organizations. Steps taken in this process were content validity, exploratory factor analysis, construction validity, reliability and detailed item analysis.
\end{abstract}

Keywords: survey research; questionnaire internal validity; ISO 9001:2000

\title{
REFERÊNCIAS
}

ABNT. Coletânea de normas de sistemas de gestão da qualidade, Associação Brasileira de Normas Técnicas. Rio de Janeiro: ABNT, 2001.

AHIRE, S. L., GOLHAR, D. Y., WALLER, M. A. Development and validation of TQM implementation constructs. Decision Sciences, v.27, n.1, p.23-56, 1996.

BAGOZZI, R.P., Yi, Y., PHILLIPS, L.W. Assessing construct validity in organizational research. Administrative Science Quarterly, V.36, n.3, p.421-59, 1991.

BLACK, S.A., PORTER, L.J. Identification of the critical factors of TQM. Decision Sciences Journal, v.27, n.1, p.01-21, 1996.

BUTTLE, F. ISO 9000: marketing motivations and benefits. International Journal of Quality \& Reliability Management, v.14, n.9, p.936-947, 1997.

CHOW-CHUA, C., GOH, M. and WAN, T.B. Does ISO 9000 certification improve business performance?. International Journal of Quality \& Reliability Management, v.20, n.8, p.936-953, 2003.

CONCA, F.J, LLOPIS, J., TARÍ, J.J. Development of a measure to assess quality management in certified firms. European Journal of Operational Research, v.156, n.3, p.683-697, 2004.

CORTINA, J.M. What is coefficient alpha? An examination of theory and applications. Journal of Applied Psychology, v.78, n.1, p.98-104, 1993.

CRONBACH, L.J., MEEHL, P.E. Construct validity in psychological tests. Psychological Bulletin, v.52, n.4, p.281-302, 1995. 
ESCANCIANO, C., FERNÁNDEZ, E., VÁZQUEZ, C. Influence of ISO 9000 certification on the progress of Spanish industry towards TQM. International Journal of Quality \& Reliability Management, v.18, n.5, p.481-494, 2001.

GIL, A.C. Métodos e técnicas de pesquisa social. 4. ed. São Paulo: Atlas, 1995.

GIUFFRE, M. Designing research survey design part one. Journal of PeriAnesthesia Nursing, v.12, n.4, p.275-80, 1997 .

GIUFFRE, M. Designing research survey design part two. Journal of PeriAnesthesia Nursing, v.12, n.5, p.358-362, 1997b.

GOTZAMANI, K., TSIOTRAS, G. The true motives behind ISO 9000 certification: their effect on the overall certification benefits and long-term contribution towards TQM. International Journal of Quality \& Reliability Management, v.19, n.2, p.151-169, 2002.

HAYNES, S. N., RICHARD, D. C. S., KUBANY, E. S. Content validity in psychological assessment: a functional approach to concepts and methods. Psychological Assessment, v.7, n.3, p.238-247, 1995.

HAIR, J., ANDERSON, R.E., TATHAM, R.L., BLACK, W.C. Multivariate data analysis. 4th ed. Englewood Cliffs: Prentice Hall, 1995.

HÄVERSJÖ, T. The financial effects of ISO 9000 registration for Danish companies. Managerial Auditing Journal, v.15, n.1/2, p.47-52, 2000.

HERAS, I., CASADESÚS, M., DICK, G. P. M. ISO 9000 registration's impact on sales and profitability: a longitudinal analysis of performance before and after accreditation. International Journal of Quality \& Reliability Management, v.19, n.6, p.774-791, 2002a.

HERAS, I., CASADESÚS, M., DICK, G. P. M. ISO 9000 certification and the bottom line: a comparative study of the profitability of Basque region firms. Managerial Auditing Journal, v.17, n.1/2, p.72-78, 2002b.

HUBLEY, A. M., ZUMBO, B. D. A dialectic on validity: where we have been and where we are going. Journal of General Psychology, v. 23, n.3, p.207-215, 1996.

ISO. The ISO survey 2007, International Organization for Standardization. Genebra: ISO, 2007. Disponível em: <http://www.iso.org/iso/survey2007.pdf >. Acesso em: 22 dez. 2009.

JOHNSON, R. A., WICHERN, D. W. Applied multivariate analysis. Englewood Cliffs: Prentice Hall, 1982.

KAYNAK, $\mathrm{H}$. The relationship between total quality management practices and their effects on firm performance. Journal of Operations Management, v. 21, n.4, p.405435, 2003. 
LEUNG, H.K.N., CHAN, K.C.C., Lee, T.Y. Costs and benefits of ISO 9000 series: a practical study. International Journal of Quality \& Reliability Management, v. 16, n.7, p.675-691, 1999.

NAVEH, E., MARCUS, A. Achieving competitive advantage through implementing a replicable management standard: installing and using ISO 9000. Journal of Operations Management, v.24, n.1, p.1-26, 2005.

NQA. NQA guide to certification, National Quality Assurance. Disponível em: <http://www.nqa.com/guide12a.html>. Acesso em: 05 out. 2006.

NUNNALLY, J.C. 1978. Psychometric theory, 2nd ed., McGraw-Hill, New York.

PETERSON, R.A. A meta-analysis of Cronbach's coefficient alpha. Journal of Consumer Research, v.21, n.2, p.381-391, 1994.

POWELL, T.C. Total quality management as competitive advantage: a review and empirical study. Strategic Management Journal, v.16, n.1, p.15-37, 1995.

RAHMAN, S. A comparative study of TQM practice and organisational performance of SMEs with and without ISO 9000 certification. International Journal of Quality \& Reliability Management, v.18, n.1, p.35-49, 2001.

SARAPH, J.V., BENSON, P.G., SCHROEDER, R.G. An instrument for measuring the critical factors of quality management. Decision Sciences, v. 20, n.4, p.10-829, 1989.

SINGELS, J., RUËL, G., WATER, H.V. ISO 9000 series certification and performance. International Journal of Quality \& Reliability Management, v.18, n.1, p.62-75, 2001.

SOHAL, A., TERZIOVSKI, M. TQM in Australia: factors critical to success. International Journal of Quality \& Reliability Management, v.17, n. 2, p.158-167, 2000.

TERLAAK, A, KING, A.A. The effect of certification with the ISO 9000 quality management standard: a signaling approach. Journal of Economic Behavior \& Organization, v.60, n.4, p.579-602, 2005.

TERZIOVSKI, M., SAMSON, D., DOW, D. The business value of quality management systems certification: evidence from Australia and New Zealand. Journal of Operations Management, v.15, n.1, p.1-18, 1997.

WILSON, D., COLLIER, D. A. An empirical investigation of the Malcolm Baldrige National Quality Award causal model. Decision Sciences, v.31, n.2, p.361-390, 2000. 